\title{
Sauerstoff mit hoher Flussrate über Tracheostoma erleichtert Weaning nach Langzeitbeatmung bei Patienten mit restriktiver Lungenfunktionsstörung: zwei Fallberichte
}

\section{Chieko Mitaka Masahiko Odoh Daizoh Satoh Tadasuke Hashiguchi Eiichi Inada}

${ }^{a}$ Department of Anesthesiology and Pain Medicine, Juntendo University, Tokio, Japan;

${ }^{b}$ Department of Esophageal and Gastroentereological Surgery, Juntendo University, Tokio, Japan

Schlüsselwörter

Sauerstoff mit hoher Flussrate · Hyperkapnie .

Restriktive Lungenfunktionsstörung · Tracheostoma · Weaning

\section{Zusammenfassung}

Hintergrund: Die Entwöhnung von einer längerfristigen maschinellen Beatmung bei tracheotomierten Patienten mit restriktiver Lungenfunktionsstörung ist extrem schwierig. Eine Sauerstoffgabe mit hoher Flussrate über das Tracheostoma liefert erwärmtes und befeuchtetes Sauerstoffgas mit $>10 \mathrm{l} / \mathrm{min}$. Jedoch liegen erst wenige Berichte zur Anwendung von Sauerstoff mit hoher Flussrate über Tracheostoma im Rahmen der Entwöhnung vom Beatmungsgerät bei Patienten mit restriktiver Lungenfunktionsstörung vor. Wir berichten über erfolgreiches Weaning vom Beatmungsgerät bei Patienten mit restriktiver Lungenfunktionsstörung mittels Sauerstoffgabe mit hoher Flussrate über Tracheostoma.

Vorstellung der Fälle: Der erste Patient ist ein 78-jähriger Japaner mit schwerer Pneumokokken-Pneumonie, der nach Ösophagektomie wegen eines Ösophaguskarzinoms mehr als einen Monat lang beatmet wurde. Nach einer Tracheotomie wegen der längerfristigen maschinellen Beatmung entwickelte sich eine restriktive Lungenfunktionsstörung mit einem Atemzugvolumen von 230-240 $\mathrm{ml}$ und einer statischen Compliance von $14-15 \mathrm{ml} / \mathrm{cm} \mathrm{H}_{2} \mathrm{O}$ mit 10

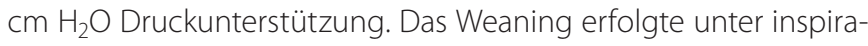
torischer Unterstützung mit Sauerstoff mit hoher Flussrate über Tracheostoma über einen Zeitraum von 16 Tagen (Flussrate von 40 I/min und inspiratorische Sauerstofffraktion von 0,25). Der zweite
Patient ist ein 69-jähriger Japaner, bei dem nach Ösophagektomie eine Aspirationspneumonie auftrat und der längerfristig über Tracheostoma beatmet wurde. Er entwickelte eine restriktive Lungenfunktionsstörung. Die Sauerstoffgabe mit hoher Flussrate über das Tracheostoma (Flussrate $40 \mathrm{l} / \mathrm{min}$ und inspiratorische Sauerstofffraktion von 0,25) erfolgte unter Messung des Atemwegsdrucks am Eingang des Tracheotomietubus. Folgende Werte wurden gemessen: 0,21-0,3 cm H $\mathrm{H}_{2} \mathrm{O}, 0,21-0,56 \mathrm{~cm} \mathrm{H}_{2} \mathrm{O}, 0,54-0,91 \mathrm{~cm} \mathrm{H} \mathrm{H}_{2} \mathrm{O}, 0,76-$ $2,01 \mathrm{~cm} \mathrm{H}_{2} \mathrm{O}, 1,17-2,01 \mathrm{~cm} \mathrm{H}_{2} \mathrm{O}$ und 1,76-2,01 $\mathrm{cm} \mathrm{H}_{2} \mathrm{O}$ bei $10 \mathrm{l} / \mathrm{min}$, $20 \mathrm{l} / \mathrm{min}, 30 \mathrm{l} / \mathrm{min}, 40 \mathrm{l} / \mathrm{min}, 50 \mathrm{l} / \mathrm{min} \mathrm{bzw} .60 \mathrm{l} / \mathrm{min}$. Der Atemwegsdruck war kontinuierlich positiv und wurde auch während der Inspiration nicht negativ, was darauf hindeutet, dass die Sauerstoffgabe mit hoher Flussrate über Tracheostoma die Inspirationsanstrengung verringert. Das Weaning erfolgte unter inspiratorischer Unterstützung mit Sauerstoff mit hoher Flussrate über Tracheostoma über einen Zeitraum von 12 Tagen.

Schlussfolgerungen: Die Sauerstoffgabe mit hoher Flussrate über Tracheostoma kann mit der Sauerstoffzufuhr die Inspirationsanstrengung verringern und das Atemzugvolumen vergrößern und so das Weaning nach Langzeitbeatmung bei Patienten mit restriktiver Lungenfunktionsstörung erleichtern.

(c) 2018 Der Autor/die Autoren

\section{KARGER}

Fax +4976145207 14

information@karger.com

www.karger.com
() 2019 S. Karger GmbH, Freiburg

Accessible online at:

www.karger.com/kkp
Chieko Mitaka, MD, PhD

Department of Anesthesiology and Pain Medicine Juntendo University

2-1-1, Hongo, Bunkyo-ku, Tokio 113-8421, Japan mitaka@juntendo.ac.jp 


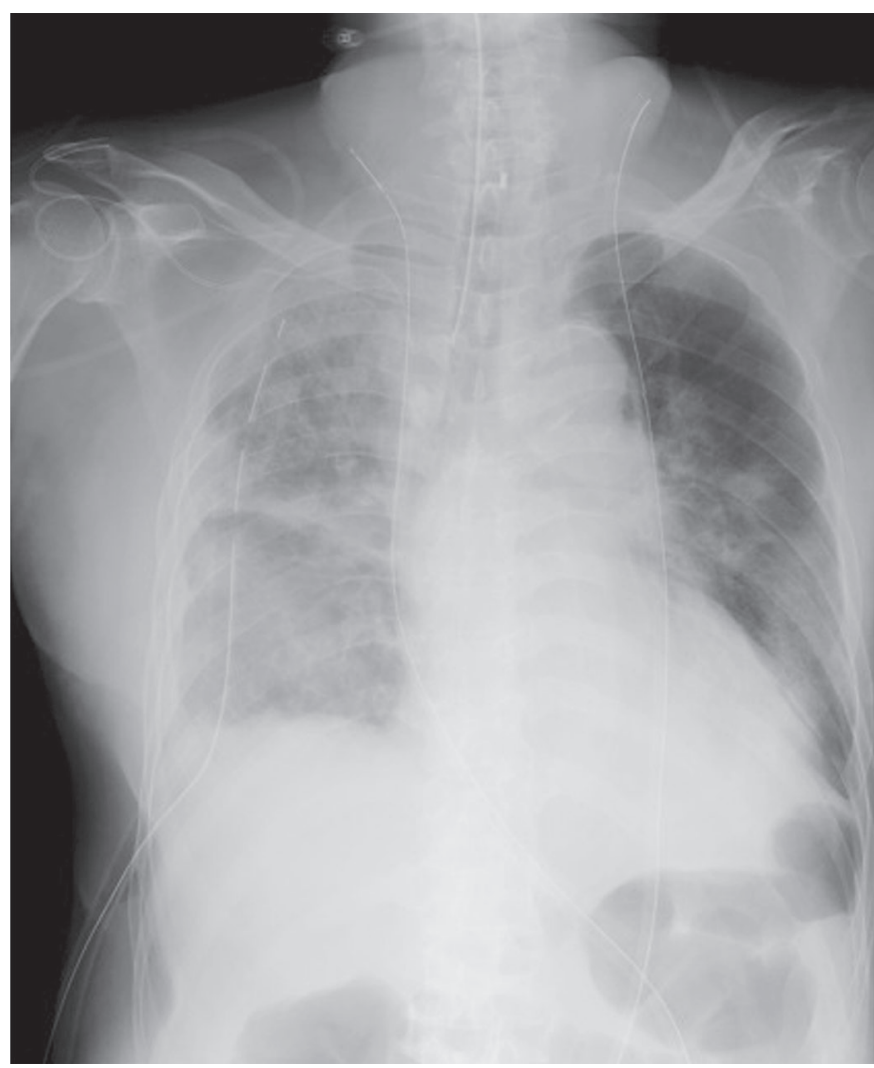

Abb. 1. Diffuse bilaterale Lungeninfiltrate, vorwiegend in der rechten Lunge, mit Pleuraerguss im Thoraxröntgen nach Tracheaintubation an Tag 5 auf der Intensivstation.

\section{Hintergrund}

Die Entwöhnung von einer längerfristigen maschinellen Beatmung bei tracheotomierten Patienten mit restriktiver Lungenfunktionsstörung ist extrem schwierig. Eine Beatmungsdauer von mehr als 21 Tagen ist mit erhöhter Mortalität, höherem Versorgungsaufwand und höheren Kosten für das Gesundheitswesen verbunden [1]. Die Sauerstoffgabe mit hoher Flussrate über Tracheostoma (high-flow oxygen via tracheostomy, HFT), durchgeführt mit dem Optiflow ${ }^{\mathrm{TM}}$ (OPT 870; Fisher \& Paykel Healthcare Ltd, Auckland, Neuseeland), ist ein Verfahren zur Bereitstellung von erwärmtem und befeuchtetem Sauerstoffgas mit einer Rate von $>10$ l/min. Obwohl auch die Sauerstoffgabe mit hoher Flussrate über Nasensonde (high-flow oxygen through nasal cannula, HFNC) klinischen Nutzen gezeigt hat, zum Beispiel in Form verbesserter Oxygenierung [2] und geringerer 90-Tages-Mortalität [3], unterscheiden sich die Mechanismen der HFT möglicherweise von denen der HFNC. Darüber hinaus liegen erst sehr wenige Daten zur Anwendung von HFT im Rahmen der Entwöhnung vom Beatmungsgerät bei Patienten mit restriktiver Lungenfunktionsstörung vor. Unsere Hypothese ist, dass die HFT das Atemzugvolumen bei Patienten mit restriktiver Lungenfunktionsstörung erhöht, indem die Inspirationsanstrengung reduziert wird. Wir berichten hier über zwei Fälle, in denen die Atemunterstützung mit HFT beim Weaning nach längerfristiger maschineller
Beatmung bei Patienten mit restriktiver Lungenfunktionsstörung Nutzen gezeigt hat.

\section{Vorstellung der Fälle}

\section{Fall 1}

Ein 78-jähriger Japaner (Größe: 163,3 cm, Gewicht: 61,3 kg) kam nach Ösophagektomie mit Magenrekonstruktion wegen eines Ösophaguskarzinoms auf unsere Intensivstation. Seine Körpertemperatur betrug $36,9^{\circ} \mathrm{C}$ und die Herzfrequenz 96 Schläge/min. Bei der Aufnahme auf die Intensivstation betrug sein Blutdruck 148/68 mm Hg. Die körperliche und neurologische Untersuchung war nicht signifikant. Die Vorgeschichte umfasste eine Appendektomie im Alter von 8 Jahren und eine lumbale Spinalkanalstenose im Alter von 70 Jahren. Er rauchte keine Zigaretten, aber trank japanischen Alkohol (360 ml/Tag). In der Familienanamnese gab es einen älteren Bruder, der an Speiseröhrenkrebs verstorben war.

An Tag 5 auf der Intensivstation trat Fieber bis $38,0{ }^{\circ} \mathrm{C}$ neu auf, außerdem vermehrtes Sputum, Hypoxämie $(90 \%$ arterielle Sauerstoffsättigung $\left(\mathrm{SpO}_{2}\right)$ laut Pulsoximetrie unter Masken-Sauerstoffgabe mit 10 l/min) und bilaterale grobe Rasselgeräusche. Daher wurde der Patient intubiert. Ein Thoraxröntgen ergab diffuse bilaterale Lungeninfiltrate, vorwiegend in der rechten Lunge, mit Pleuraerguss (Abb. 1). Die Laboruntersuchungen ergaben folgen-

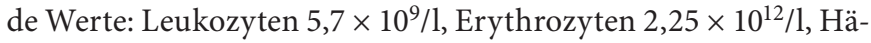
moglobin 7,3 g/dl, Hämatokrit 21,4\%, Thrombozyten $145 \times 10^{9} / 1$, Aspartataminotransferase $54 \mathrm{U} / \mathrm{l}$, Alaninaminotransferase 55 U/l, Gesamtbilirubin 4,11 mg/dl, Albumin 2,2 g/dl, HarnstoffStickstoff $38 \mathrm{mg} / \mathrm{dl}$, Kreatinin 0,83 mg/dl, C-reaktives Protein $18,7 \mathrm{mg} / \mathrm{dl}$ und Urin-Kreatinin $95 \mathrm{mg} / \mathrm{dl}$ nach Intubation. Da die Sputumkultur an Tag 7 auf der Intensivstation Streptococcus pneumoniae ergab, wurde das Antibiotikum von Cefmetazol auf Meropenem umgestellt.

Der Patient wurde über lange Zeit maschinell beatmet und schließlich an Tag 38 auf der Intensivstation einer Tracheotomie unterzogen. Obwohl die Oxygenierung mit einem arteriellen Sauerstoffpartialdruck/inspiratorische Sauerstofffraktion $\left(\mathrm{PaO}_{2} / \mathrm{F}_{\mathrm{I}} \mathrm{O}_{2}\right)$ von $>300 \mathrm{~mm} \mathrm{Hg}$ gut war, entwickelte er eine restriktive Lungenfunktionsstörung: Atemzugvolumen 230-240 ml, statische Compliance $14-15 \mathrm{ml} / \mathrm{cm} \mathrm{H}_{2} \mathrm{O}$ bei Druckunterstützungsbeatmung mit 10 $\mathrm{cm} \mathrm{H}_{2} \mathrm{O}$, Atemfrequenz 34 Züge/min und arterieller Kohlendioxidpartialdruck $\left(\mathrm{PaCO}_{2}\right) 46 \mathrm{~mm} \mathrm{Hg}$. Wir versuchten ein Weaning von der Beatmung mit Unterstützung durch HFT mit einer Flussrate von $40 \mathrm{l} / \mathrm{min}$ und einer $\mathrm{F}_{\mathrm{I}} \mathrm{O}_{2}$ von 0,25 , da der maximale inspiratorische Fluss des Beatmungsgeräts 40 1/min bei Druckunterstützungsbeatmung mit $10 \mathrm{~cm} \mathrm{H}_{2} \mathrm{O}$ betrug. Durch Einstellung derselben Flussrate wie am Beatmungsgerät hofften wir, die Inspirationsanstrengung zu reduzieren. In den ersten 8 Tagen wurde tagsüber die HFT angewendet und nachts ein Beatmungsgerät mit $5 \mathrm{~cm} \mathrm{H}_{2} \mathrm{O}$ Druckunterstützung mit einem positiven endexspiratorischen Druck (positive end-expiratory pressure, PEEP) von $5 \mathrm{~cm}$ $\mathrm{H}_{2} \mathrm{O}$. In den folgenden 8 Tagen wurde rund um die Uhr die HFT angewendet. In Tabelle 1 sind die Atmungsparameter während der HFT aufgeführt. Unter Atemwegsunterstützung mit HFT war der 
Tab. 1. Veränderungen der Atmungsparameter unter Sauerstoffgabe mit hoher Flussrate über Tracheostoma in Fall 1

\begin{tabular}{|c|c|c|c|c|c|c|c|c|c|c|c|c|c|c|c|c|}
\hline HFT-Tag & 1 & 2 & 3 & 4 & 5 & 6 & 7 & 8 & 9 & 10 & 11 & 12 & 13 & 14 & 15 & 16 \\
\hline \multicolumn{17}{|l|}{ HFT-Einstellungen } \\
\hline $\mathrm{F}_{1} \mathrm{O}_{2}$ & 0,25 & 0,25 & 0,25 & 0,25 & 0,25 & 0,25 & 0,25 & 0,25 & 0,25 & 0,25 & 0,25 & 0,25 & 0,25 & 0,25 & 0,25 & 0,25 \\
\hline Flussrate (I/Minute) & 40 & 40 & 40 & 40 & 40 & 40 & 40 & 20 & 15 & 15 & 15 & 15 & 15 & 15 & 15 & 15 \\
\hline Atemfrequenz (Züge/Minute) & 40 & 30 & 35 & 37 & 26 & 30 & 35 & 36 & 35 & 27 & 29 & 26 & 22 & 20 & 22 & 21 \\
\hline $\mathrm{SpO}_{2}(\%)$ & 97 & 98 & 97 & 97 & 96 & 98 & 97 & 98 & 96 & 96 & 96 & 96 & 98 & 95 & 98 & 96 \\
\hline $\mathrm{etCO}_{2}(\mathrm{mmHg})$ & 47 & 41 & 40 & 39 & 42 & 42 & 40 & 41 & 39 & 35 & 34 & 35 & 37 & 35 & 38 & 41 \\
\hline $\mathrm{V}_{\mathrm{T}}(\mathrm{ml})$ & & & & 240 & & & & 260 & & & 300 & & & 300 & & \\
\hline \multicolumn{17}{|l|}{ Arterielle Blutgaswerte } \\
\hline $\mathrm{pH}$ & & & & 7,46 & & & & & & & 7,47 & & & & & \\
\hline $\mathrm{PaCO}_{2}(\mathrm{~mm} \mathrm{Hg})$ & & & & 47 & & & & & & & 44 & & & & & \\
\hline $\mathrm{PaO}_{2}(\mathrm{~mm} \mathrm{Hg})$ & & & & 92 & & & & & & & 87 & & & & & \\
\hline $\mathrm{HCO}_{3-}(\mathrm{mmol} / \mathrm{l})$ & & & & 32,5 & & & & & & & 31,2 & & & & & \\
\hline
\end{tabular}

Abkürzungen: et $\mathrm{CO}_{2}$ endexspiratorisches Kohlendioxid, $\mathrm{FIO}_{2}$ inspiratorische Sauerstofffraktion, HFT Sauerstoff mit hoher Flussrate über Tracheostoma, $\mathrm{HCO}$ Bicarbonation, $\mathrm{PaCO}_{2}$ arterieller Kohlendioxidpartialdruck, $\mathrm{PaO}_{2}$ arterieller Sauerstoffpartialdruck, SpO 2 arterielle Sauerstoffsättigung laut Pulsoximetrie, VT Atemzugvolumen laut Wright-Spirometer

Zustand des Patienten stabil, und die physiotherapeutische Rehabilitation verlief ohne Zwischenfälle. Die HFT lieferte optimal befeuchtetes Gas, was zu flüssigerem Bronchialsekret beitrug. An Tag 127 wurde er von der Intensiv- auf die Normalstation entlassen. Seine physiotherapeutische Rehabilitation wurde weitere zwei Monate fortgeführt, und an Tag 201 des Krankenhausaufenthalts wurde er in eine Rehabilitationsklinik verlegt.

\section{Fall 2}

Ein 69-jähriger Japaner (Größe: 160,0 cm, Gewicht: 37,1 kg) kam nach einem Besuch der Notaufnahme wegen schwerer Dyspnoe auf unsere Intensivstation. Seine Vorgeschichte umfasste eine extrakorporale Stoßwellenlithotripsie wegen Harnsteinen (56 Jahre), eine endoskopische Polypektomie von Kolonpolypen (66 Jahre) und eine Ösophagektomie mit Magenrekonstruktion nach präoperativer Radiochemotherapie wegen eines Ösophaguskarzinoms (66 Jahre). Er rauchte seit 40 Jahren 30 Zigaretten pro Tag und trank Whisky (1 Flasche/4 Tage). Er wandte keinerlei Medikamente an. In der Familienanamnese war eine Großmutter an Leberkrebs verstorben. Bei der körperlichen Untersuchung war er verwirrt und ruhelos, die Atmung war flach mit reduzierter Luftzufuhr in beide Lungenflügel. Die neurologische Untersuchung war nicht signifikant. Folgende Vitalzeichen wurden gemessen: Herzfrequenz 122 Schläge/min, Blutdruck 80/58 mm Hg, Atemfrequenz 26 Züge/min, Körpertemperatur $38,7^{\circ} \mathrm{C}$. Die arterielle Blutgasanalyse ergab eine respiratorische Azidose: $\mathrm{pH} 7,21, \mathrm{PaCO}_{2}$ $117 \mathrm{~mm} \mathrm{Hg}, \mathrm{PaO}_{2} 76 \mathrm{~mm} \mathrm{Hg}$ und Bicarbonationen $\left(\mathrm{HCO}_{3}{ }^{-}\right)$45,9 $\mathrm{mmol} / \mathrm{l}$ unter Masken-Sauerstoffgabe mit $61 / \mathrm{min}$. Selbst mit einer Beutel-Ventil-Maske konnte keine ausreichende Beatmung erreicht werden. Der Patient wurde unverzüglich intubiert, und zahlreiche Speisepartikel wie Bohnen und Reis wurden aus der Luftröhre aspiriert. Daher wurden so schnell wie möglich mittels Bronchoskopie die noch verbleibenden Speisereste entfernt. Ein Thoraxröntgen ergab diffuse bilaterale Lungeninfiltrate (Abb. 2). Nachdem eine Aspirationspneumonie diagnostiziert wurde,

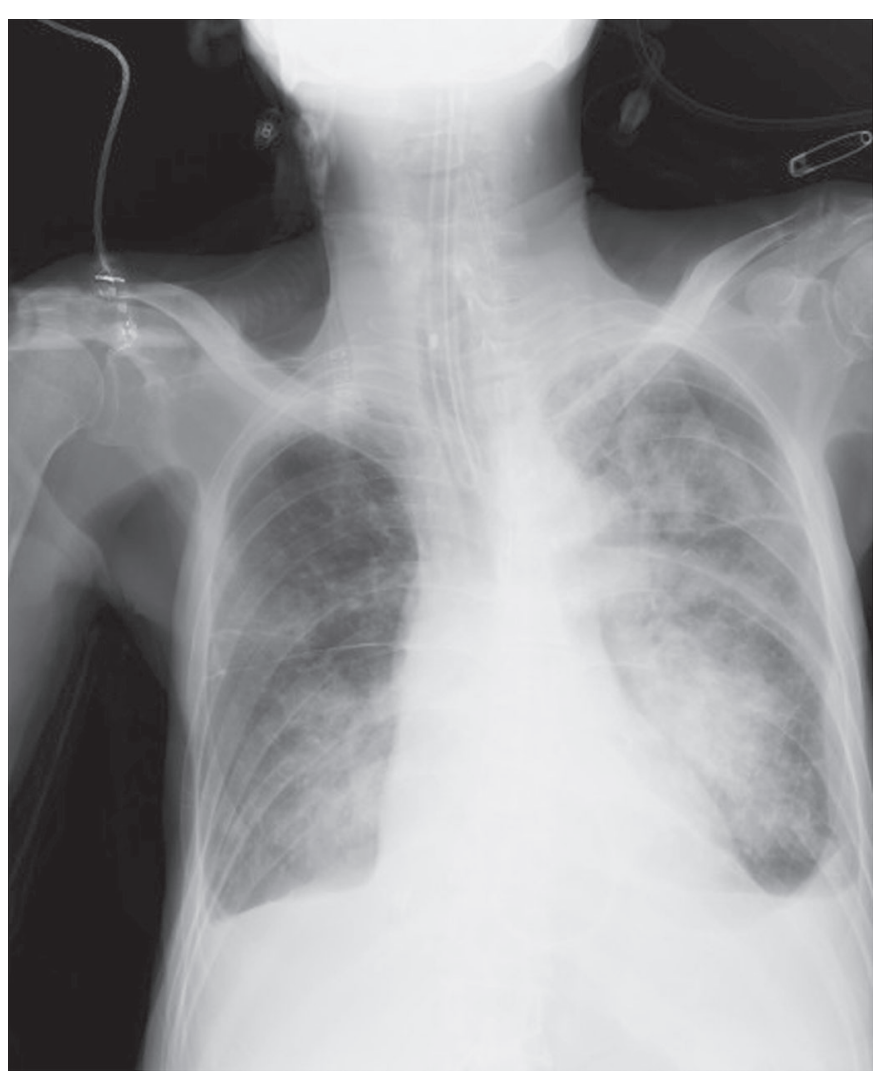

Abb. 2. Diffuse bilaterale Lungeninfiltrate im Thoraxröntgen nach Tracheaintubation an Tag 1 auf der Intensivstation.

wurde eine maschinelle Beatmung eingeleitet. Die Laboruntersuchungen ergaben folgende Werte: Leukozyten $0,9 \times 10^{9} / 1$, Erythrozyten 4,66 × 10 $12 / 1$, Hämoglobin 12,1 g/dl, Hämatokrit 41,0 \%, Thrombozyten $297 \times 10^{9} /$, Aspartataminotransferase $17 \mathrm{U} / \mathrm{l}$, Alaninaminotransferase 7 U/l, Gesamtbilirubin 0,59 mg/dl, Albumin 2,2 g/dl, Harnstoff-Stickstoff 26 mg/dl, Kreatinin 0,75 mg/ 
Tab. 2. Veränderungen der Atmungsparameter unter Sauerstoffgabe mit hoher Flussrate über Tracheostoma in Fall 2

\begin{tabular}{|c|c|c|c|c|c|c|c|c|c|c|c|c|}
\hline HFT-Tag & 1 & 2 & 3 & 4 & 5 & 6 & 7 & 8 & 9 & 10 & 11 & 12 \\
\hline \multicolumn{13}{|l|}{ HFT-Einstellungen } \\
\hline $\mathrm{F}_{1} \mathrm{O}_{2}$ & 0,3 & 0,25 & 0,25 & 0,3 & 0,3 & 0,3 & 0,3 & 0,3 & 0,3 & 0,3 & 0,3 & 0,3 \\
\hline Flussrate (I/Minute) & 40 & 40 & 40 & 40 & 40 & 50 & 40 & 40 & 40 & 40 & 30 & 20 \\
\hline Atemfrequenz (Züge/Minute) & 39 & 22 & 26 & 24 & 24 & 31 & 32 & 30 & 30 & 23 & 26 & 20 \\
\hline $\mathrm{SpO}_{2}(\%)$ & 96 & 94 & 95 & 94 & 95 & 96 & 97 & 97 & 98 & 98 & 99 & 100 \\
\hline $\mathrm{etCO}_{2}(\mathrm{mmHg})$ & 44 & 37 & 40 & 42 & 46 & 48 & 46 & 49 & 48 & 45 & 43 & 46 \\
\hline $\mathrm{V}_{\top}(\mathrm{ml})$ & & & & 220 & 210 & 210 & 230 & 220 & 220 & 230 & 230 & 230 \\
\hline \multicolumn{13}{|l|}{ Arterielle Blutgaswerte } \\
\hline $\mathrm{pH}$ & & 7,47 & 7,43 & 7,41 & 7,37 & 7,40 & 7,38 & 7,39 & 7,42 & 7,42 & 7,42 & 7,42 \\
\hline $\mathrm{PaCO}_{2}(\mathrm{~mm} \mathrm{Hg})$ & & 50 & 53 & 58 & 68 & 66 & 70 & 66 & 61 & 59 & 59 & 60 \\
\hline $\mathrm{PaO}_{2}(\mathrm{~mm} \mathrm{Hg})$ & & 61 & 73 & 68 & 77 & 80 & 86 & 79 & 91 & 93 & 72 & 85 \\
\hline $\mathrm{HCO}_{3-}(\mathrm{mmol} / \mathrm{l})$ & & 53,1 & 34,7 & 35,9 & 38,5 & 40,1 & 40,1 & 38,8 & 39,1 & 37,9 & 36,9 & 37,8 \\
\hline
\end{tabular}

Abkürzungen: et $\mathrm{CO}_{2}$ endexspiratorisches Kohlendioxid, $\mathrm{FIO}_{2}$ inspiratorische Sauerstofffraktion, HFT Sauerstoff mit hoher Flussrate über Tracheostoma, $\mathrm{HCO}$ Bicarbonation, $\mathrm{PaCO}_{2}$ arterieller Kohlendioxidpartialdruck, $\mathrm{PaO}_{2}$ arterieller Sauerstoffpartialdruck, SpO $\mathrm{O}_{2}$ arterielle Sauerstoffsättigung laut Pulsoximetrie, VT Atemzugvolumen (laut Wright-Spirometer)

dl und C-reaktives Protein 1,7 mg/dl bei Aufnahme auf die Intensivstation.

An Tag 32 auf der Intensivstation wurde der Patient wegen der langen Beatmungsdauer einer Tracheotomie unterzogen. Obwohl die Oxygenierung gut war $\left(\mathrm{PaO}_{2} / \mathrm{F}_{\mathrm{I}} \mathrm{O}_{2}>300 \mathrm{~mm} \mathrm{Hg}\right)$, entwickelte er eine restriktive Lungenfunktionsstörung: Atemzugvolumen 210$220 \mathrm{ml}$, statische Compliance $16-17 \mathrm{ml} / \mathrm{cm} \mathrm{H}_{2} \mathrm{O}$ bei Druckunterstützungsbeatmung mit $10 \mathrm{~cm} \mathrm{H}_{2} \mathrm{O}$, Atemfrequenz $30 \mathrm{Züge/min}$ und $\mathrm{PaCO}_{2} 46 \mathrm{~mm} \mathrm{Hg}$. Wir versuchten ein Weaning von der Beatmung mit Unterstützung durch HFT (Flussrate $40 \mathrm{l} / \mathrm{min}, \mathrm{F}_{\mathrm{I}} \mathrm{O}_{2}$ 0,25). In Tabelle 2 sind die Atmungsparameter während der 4-tägigen HFT aufgeführt. An Tag 4 der HFT wurden folgende arteriellen Blutgaswerte gemessen: $\mathrm{pH} 7,41, \mathrm{PaCO}_{2} 58 \mathrm{~mm} \mathrm{Hg}, \mathrm{PaO}_{2} 68$ $\mathrm{mm} \mathrm{Hg}$ und $\mathrm{HCO}_{3}{ }^{-} 35,7 \mathrm{mmol} / \mathrm{l}$. Wir beurteilten seinen Zustand als zufriedenstellend und wechselten von der HFT zur Sauerstoffgabe über ein T-Stück mit 3 1/min. Eine Stunde nach Beginn dieser Sauerstoffgabe klagte er über Dyspnoe, und die arteriellen Blutgase verschlechterten sich moderat ( $\mathrm{pH}$ 7,34, $\mathrm{PaCO}_{2} 72 \mathrm{~mm} \mathrm{Hg}, \mathrm{PaO}_{2}$ $106 \mathrm{~mm} \mathrm{Hg}$ und $\left.\mathrm{HCO}_{3}^{-} 37,3 \mathrm{mmol} / \mathrm{l}\right)$. Das Atemzugvolumen betrug zu diesem Zeitpunkt $200 \mathrm{ml}$. Wir entschieden uns, wieder zur HFT zu wechseln (Flussrate $40 \mathrm{l} / \mathrm{min}, \mathrm{F}_{\mathrm{I}} \mathrm{O}_{2}$ 0,25), und 1 Stunde später hatten sich die Blutgaswerte verbessert: $\mathrm{pH} 7,40, \mathrm{PaCO}_{2} 60$ $\mathrm{mm} \mathrm{Hg}, \mathrm{PaO}_{2} 71 \mathrm{~mm} \mathrm{Hg}$ und $\mathrm{HCO}_{3}{ }^{-} 35,9 \mathrm{mmol} / \mathrm{l}$. An Tag 5 der HFT stieg der $\mathrm{PaCO}_{2}$ auf $70 \mathrm{~mm} \mathrm{Hg}$. Dieser Umstand deutete auf eine Ermüdung der Atemmuskulatur hin, jedoch waren keine Dyspnoebeschwerden festzustellen. Wir beschlossen, die HFT nun tagsüber anzuwenden und nachts auf ein Beatmungsgerät $(10 \mathrm{~cm}$ $\mathrm{H}_{2} \mathrm{O}$ Druckunterstützung mit $5 \mathrm{~cm} \mathrm{H}_{2} \mathrm{O}$ PEEP) umzustellen; dieses Behandlungsschema wurde für die nächsten 8 Tage angewandt. Wir beurteilten den Trachealdruck während der HFT mit einem Durchflussanalysator $\left(\right.$ CITREX $^{\circledR}$, TOKIBO Co. Ltd., Tokio, Japan) zur Messung des Atemwegsdrucks am Eingang des Tracheotomietubus. Folgende Werte wurden gemessen: 0,21-0,3 $\mathrm{cm} \mathrm{H}_{2} \mathrm{O}$, 0,21-0,56 cm H $\mathrm{cm}_{2} \mathrm{O}, 0,54-0,91 \mathrm{~cm} \mathrm{H}_{2} \mathrm{O}, 0,76-2,01 \mathrm{~cm} \mathrm{H}_{2} \mathrm{O}, 1,17-$ 2,01 $\mathrm{cm} \mathrm{H}_{2} \mathrm{O}$ und 1,76-2,01 $\mathrm{cm} \mathrm{H}_{2} \mathrm{O}$ bei $10 \mathrm{l} / \mathrm{min}, 20 \mathrm{l} / \mathrm{min}, 30 \mathrm{l} /$ min, 40 l/min, 50 l/min bzw. 60 l/min. Der Atemwegsdruck war kontinuierlich positiv und wurde auch während der Inspiration nicht negativ. Diese Ergebnisse deuten darauf hin, dass die HFT die Inspirationsanstrengung verringert. Unter Atmungsunterstützung mit HFT und physiotherapeutischer Rehabilitation konnte der Patient erfolgreich vom Beatmungsgerät entwöhnt werden. An Tag 51 wurde er von der Intensiv- auf die Normalstation entlassen. Die physiotherapeutische Rehabilitation wurde einen weiteren Monat fortgeführt. An Tag 86 seines Krankenhausaufenthalts wurde der Patient nach Hause entlassen und kam zu einer Kontrolluntersuchung zurück.

\section{Diskussion}

Wir beschreiben hier nach bestem Wissen den ersten Versuch, bei Patienten mit restriktiver Lungenfunktionsstörung HFT-Unterstützung zum Weaning nach längerfristiger Beatmung einzusetzen. Unsere Daten deuten darauf hin, dass die HFT für die Entwöhnung vom Beatmungsgerät von Nutzen war. Bei den beiden Patienten, die wir von der Beatmung entwöhnten, lag eine ausgeprägte Restriktion der Atemmuskulatur vor. Die HFT steigerte die alveoläre Ventilation durch Erhöhung des Atemzugvolumens und bewirkte so eine Reduktion der Atemfrequenz und des $\mathrm{PaCO}_{2}$. Die Atmungsunterstützung mittels HFT könnte auch für Patienten von Nutzen sein, deren Atemzugvolumen wegen Tachypnoe oder schneller oberflächlicher Atmung herabgesetzt ist. Beim zweiten hier vorgestellten Patienten stieg der $\mathrm{PaCO}_{2}$ von 58 auf $72 \mathrm{~mm} \mathrm{Hg}$, nachdem er von einer HFT auf ein T-Stück umgestellt wurde. Nach der Rückkehr zur HFT ging der $\mathrm{PaCO}_{2}$ auf den vorherigen Wert zurück. Die HFT-Flussrate wurde auf denselben Wert wie das Beatmungsgerät eingestellt wurde, und es wurde schon von einer leichten Reduktion der Inspirationsanstrengung eine Erhöhung des Atemzugvolumens erwartet. Der Hauptmechanismus der HFT bei Patienten mit restriktiver Lungenfunktionsstörung beruht unserer Ansicht nach auf einer solchen Verrin- 
gerung der Inspirationsanstrengung und Erhöhung des Atemzugvolumens. Insofern erwarteten wir, dass die Atemarbeit des Patienten sich durch die langsamere und tiefere Atmung verringern würde. Die HFT kann mit ihrer hohen Flussrate die Inspiration von Patienten mit restriktiver Lungenfunktionsstörung unterstützen und eine wichtige Rolle bei der Verringerung der Atemarbeit spielen. In einem randomisierten Crossover-Vergleich zwischen HFT und T-Stück von Corley et al. [4] war der mittlere Atemwegsdruck 15 min nach Beginn der HFT signifikant höher als mit dem T-Stück. Beim endexspiratorischen Lungenvolumen (EELV) ergab die Studie jedoch keinen signifikanten Unterschied zwischen HFT und dem T-Stück. Im Gegensatz zu unseren Fällen hatten die Patienten in dieser Studie keine restriktive Lungenfunktionsstörung. Die nicht vorhandene Funktionsstörung könnte erklären, warum mit HFT und T-Stück ähnliche EELVWerte gemessen wurden. In unseren Fällen nahmen $\mathrm{PaCO}_{2}$ und Atemfrequenz unter der HFT allmählich ab, während das Atemzugvolumen allmählich zunahm.

Die HFNC befreit die oberen Atemwege von Exspirationsluft, verringert den Totraum $[5,6]$ und reduziert zugleich die Atemarbeit und den $\mathrm{PaCO}_{2}$ [7]. Das HFT-System hingegen stellt im Vergleich zur HFNC einen offeneren Kreislauf der In- und Exspiration her. Der vorliegende Fallbericht zeigt, wie die HFT durch Verringerung der Inspirationsanstrengung das Atemzugvolumen von $\mathrm{Pa}-$ tienten mit restriktiver Lungenfunktionsstörung erhöhte.

\section{Schlussfolgerungen}

Die HFT war ein wirksames Mittel für das Weaning nach langer Beatmung bei Patienten mit restriktiver Lungenfunktionsstörung. Die HFT kann mit der Sauerstoffzufuhr die Inspirationsanstrengung verringern und das Atemzugvolumen vergrößern und so die Entwöhnung vom Beatmungsgerät bei diesen Patienten erleichtern. Wir empfehlen, die Einstellungen der HFT anfänglich so zu wählen, dass sie dem maximalen inspiratorischen Fluss des Beatmungsgeräts während der Druckunterstützungs-Beatmung entsprechen, und die Dauer der HFT in Kombination mit einer physiotherapeutischen Rehabilitation graduell zu verlängern. Weitere Studien sind erforderlich, um die Wirksamkeit der HFT für das Weaning nach längerer Beatmung bei einer umfassenderen Population von Patienten mit restriktiver Lungenfunktionsstörung zu untersuchen.

\section{Danksagung}

Wir danken dem Nature Research Editing Service (http://bit.ly/NRES-HS) für die sprachliche Überarbeitung des englischen Texts.

\section{Verfügbarkeit der Daten und Materialien}

Die in dieser Arbeit verwendeten und/oder analysierten Datensätze sind auf begründete Anfrage vom Korrespondenzautor erhältlich.

\section{Beiträge der einzelnen Autoren}

CM koordinierte die Studie und trug maßgeblich zur Erstellung des Manuskripts bei. MO und TH waren verantwortlich für die Patientenversorgung und Datenerhebung. DS und EI trugen zur Interpretation der Daten bei. Alle Autoren haben die finale Fassung des Manuskripts gelesen und freigegeben.

\section{Genehmigung durch Ethikkommission und Einwilligung zur Teilnahme}

Nicht zutreffend.

\section{Zustimmung zur Veröffentlichung}

Von den Patienten wurde nach Aufklärung eine schriftliche Einverständniserklärung für die Veröffentlichung der vorliegenden Fallberichte und der dazugehörigen Bilder eingeholt. Der Wortlaut der schriftlichen Einverständniserklärung liegt zur Begutachtung durch die Chefredaktion des J Med Case Rep vor.

\section{Interessenskonflikte}

Die Autoren erklären, dass keine Interessenskonflikte bestehen.

\section{Anmerkung des Herausgebers des Originalartikels}

Springer Nature nimmt eine neutrale Position hinsichtlich rechtlicher Ansprüche in veröffentlichten Karten und institutionellen Zugehörigkeiten ein.

Eingereicht: 17. April 2018. Angenommen: 3. September 2018. Online veröffentlicht: 12. Oktober 2018.

\section{Lizenzangabe}

Chieko Mitaka, Masahiko Odoh, Daizoh Satoh, Tadasuke Hashiguchi, Eiichi Inada: High-flow oxygen via tracheostomy facilitates weaning from prolonged mechanicalventilation in patients with restrictive pulmonary dysfunction: two case reports. J Med Case Rep 2018;12:292 (https://doi. org/10.1186/s13256-018-1832-7). @The Author(s), 2018, lizensiert unter CC BY 4.0 (https://creativecommons.org/licenses/by/4.0/deed.de).

\section{Literatur}

1 Hill AD, Fowler RA, Burns KEA, et al.: Longterm outcomes and health care utilization after prolonged mechanical ventilation. Ann Am Thorac Soc 2017;14:355-362.

$\checkmark 2$ Maggiore SM, Idone FA, Vaschetto R, et al. Nasal high-flow versus Venturi mask oxygen therapy after extubation. Effects on oxygenation, comfort, and clinical outcome. Am J Respir Crit Care Med 2014;190:282-288.

$\checkmark 3$ Frat JP, Thille AW, Mercat A, et al.; for the FLORALI Study Group and the REVA Net- work: High-flow oxygen through nasal cannula in acute hypoxemic respiratory failure. $\mathrm{N}$ Engl J Med 2015;372:2185-2196.

4 Corley A, Edwards M, Spooner AJ, et al.: Highflow oxygen via tracheostomy improves oxygenation in patients weaning from mechanical ventilation: a randomized crossover study. Intensive Care Med 2017;43:465-467.

5 Fricke K, Tatkov S, Domanski U, et al.: Case report. Nasal high flow reduces hypercapnia by clearance of anatomical dead space in a COPD patient. Respir Med Case Rep 2016;19: 115-117.

6 Möller W, Feng S, Domanski U, et al.: Nasal high flow reduces dead space. J Appl Physiol 2017;122:191-197.

7 Biselli PJC, Kirkness JP, Grote L, et al.: Nasal high-flow therapy reduces work of breathing compared with oxygen during sleep in COPD and smoking controls: a prospective observational study. J Appl Physiol 2017;122: 82-88. 